\title{
Porque así soy yo. Identidad, violencias y alternativas sociales entre jóvenes pertenecientes a "barrios" o "pandillas" en colonias conflictivas de Zapopan
}

doi: http://dx.doi.org/10.32870/

espiral.v22i63.1674.g1464

J. Igor Israel González Aguirre•

La construcción de un régimen democrático en nuestros días requiere de la implementación de estrategias cada vez más innovadoras, que hagan de la hechura de las políticas un asunto verdaderamente público. Desde esta perspectiva, puede decirse que en el campo de la gobernanza contemporánea hay dos pilares fundamentales: 1) una administración pública abierta y transparente; y 2) un gobierno cada vez más próximo a la ciudadanía. Así, en la medida en que los procesos de toma de decisiones sean más incluyentes y horizontales, cercanos, la atención de las necesidades de la población será más asertiva. Si este proceso se llevara a cabo en el plano de lo local, se generarían condiciones de posibilidad para la puesta en marcha de políticas caracterizadas por un espíritu

Profesor-investigador del Departamento de Estudios sobre Movimientos Sociales del Centro Universitario de Ciencias Sociales y Humanidades de la Universidad de Guadalajara. jiigonzaleza@gmail.com emancipatorio. Un requisito para que ocurra lo anterior se encuentra en la articulación de vasos comunicantes entre las instancias productoras del saber; es decir, entre la Academia, los distintos órdenes de gobierno,

Rogelio Marcial Vázquez y Miguel Vizcarra Dávila (2014). Porque así soy yo. Identidad, violencias y alternativas sociales entre jóvenes pertenecientes a "barrios"o "pandillas" en colonias conflictivas de Zapopan. México: SEGOB/ Gobierno Municipal de Zapopan/El Colegio de Jalisco/CONFIN/Sistema Nacional de Seguridad Pública. 
y la ciudadanía. La apuesta por una labor conjunta, que vincule a los diversos actores de la sociedad en la consecución de objetivos compartidos, es una tarea fundamental de nuestra época. En este sentido, la producción de información estratégica, confiable, es un factor clave para la arquitectura de una nueva esfera pública, más deliberativa, crítica y, por ende, más democrática.

Desde esta perspectiva, el trabajo elaborado por Rogelio Marcial y Miguel Vizcarra (2014) representa un esfuerzo encaminado tanto a la generación de un diagnóstico pertinente y veraz en torno a los grupos juveniles denominados como pandillas, en el municipio de Zapopan. ¿Cómo viven? ¿A qué problemas sociales y económicos se enfrentan? ¿Cuáles son las visiones que tienen con respecto al futuro? ¿Cómo los perciben los vecinos de las colonias que habitan? ¿Cómo son vistos por algunos medios de comunicación? Estas y otras interrogantes encuentran respuesta en el documento escrito por los autores citados. No obstante, el documento no permanece en el plano analítico; es decir, no sólo reflexiona acerca de la realidad observada, también busca transformarla. En este sentido, el libro de Marcial y Vizcarra da cuenta del proceso de implementación de estrategias de intervención que apuestan por la cultura como un eje central para la producción de mejores condiciones para el desarrollo de las zonas bajo estudio y, desde luego, de la población joven-pandillera que ahí habita.

El libro está dividido en cuatro capítulos, además de un prólogo escrito por Carles Feixa, uno de los investigadores de juventud más reconocidos en todo el orbe. Además, el trabajo se acompaña de un DVD que pone de relieve tanto las minucias del proceso investigativo como el desarrollo e implementación de las estrategias de intervención resultan- 
Porque así soy yo. Identidad, violencias y alternativas sociales entre jóvenes pertenecientes a "barrios" o "pandillas" en colonias conflictivas de Zapopan

tes de la fase diagnóstica. ${ }^{1}$ De este modo, el primer capítulo se titula "Análisis y contextualización de la realidad de las juventudes en pandillas”. En este se presenta la naturaleza del trabajo, en tanto estrategia que vincula a la investigación con procesos de intervención social. Se parte del reconocimiento de la complejidad de los fenómenos sociales, sobre todo aquellos que tienen que ver con la seguridad ciudadana. Vale la pena decir en este punto que dicho tema es un elemento que atraviesa a toda la obra. De manera específica, en este primer capítulo, Marcial y Vizcarra plantean que los delitos derivados de las acciones del crimen organizado no son la única fuente de inseguridad. Los autores aseveran, más bien, que algunas de las principales problemáticas en las que se enraíza lo anterior se vinculan con la existencia de grupos juveniles en zonas urbanas pauperizadas; grupos conocidos como bandas, pandillas, o barrios. En un contexto marcado por una violencia creciente, estos grupos, en ocasiones, llevan a cabo acciones que ponen en riesgo tanto a los habitantes de sus colonias como a ellos mismos. Bajo este marco, Marcial y Vizcarra aseveran que es preciso postular la violencia como un concepto polisémico y marcado por la historicidad, por lo que no es posible comprender las expresiones concretas de este fenómeno (es decir, violencias estructurales, violencias latentes, violencias colaterales) sin una contextualización adecuada (es decir, la denominada

I. Vale la pena dar cuenta del contenido del DVD, puesto que éste tiene una importancia crucial en términos del proceso de investigación e intervención desarrollado por Marcial y Vizcarra. El disco cuenta en principio con una copia digital del libro aquí reseñado. Aunado a ello, recopila varios manuales y videos informativos que sirven como insumos para la implementación de estrategias de intervención similares a la desarrollada por los citados autores.Además, el DVD reúne algunas producciones musicales y gráficas elaboradas por los sujetos que participaron en el estudio. Finalmente, es destacable el documental titulado Donde moran los sueños, de Jonás González-Illoldi, en el que se retrata la vida de los integrantes de pandillas y las situaciones a las que estos enfrentan en los espacios en los que se desenvuelven. 
"guerra contra el crimen organizado", iniciada durante el mandato de Felipe Calderón).

Con base en estas premisas, Marcial y Vizcarra se enfocan en indagar, desde la propia mirada de los jóvenes involucrados en pandillas, algunas de las tendencias negativas a las que estos hacen frente. Ello con el objeto de diseñar acciones de intervención social encaminadas a la transformación de dichos jóvenes en "actores de paz". En última instancia, los citados autores buscan postular estrategias alternativas de reconstrucción del tejido social en las colonias marginadas bajo estudio. Vale la pena recordar que estas se encuentran en el municipio de Zapopan, y fueron elegidas con base en tres factores principales: por sus altos índices de marginación, por la presencia de pandillas, y por los problemas de violencia asociados con dichas agrupaciones. En particular, son cuatro las colonias en las que se efectuó la investigación-intervención: 1) Lomas de la Primavera; 2) Mesa de los Ocotes; 3) Santa Ana Tepetitlán; y 4) San Juan de Ocotán. No está de más señalar que en el libro se efectúa un minucioso análisis de la realidad particular de cada una de estas áreas.

En este primer capítulo también se esbozan los parámetros metodológicos que guiaron el proceso investigativo. En principio, se puso en marcha un acercamiento cuantitativo a partir de la revisión de datos estadísticos. Enseguida se abordó un eje cualitativo de corte etnográfico, cimentado en entrevistas con informantes clave y la realización de grupos de discusión. De manera específica, se buscó documentar la realidad de los jóvenes "pandilleros" que habitan en las colonias mencionadas. Lo anterior sirvió para detectar los territorios en los que se localizan las actividades (es decir, delitos y faltas administrativas) de este tipo de grupalidades. A esto se sumó tanto la identificación de las organizaciones de la sociedad civil con presencia en las zonas estudiadas, como los factores de frustración, estrés, 
Porque así soy yo. Identidad, violencias y alternativas sociales entre jóvenes pertenecientes a "barrios" o "pandillas" en colonias conflictivas de Zapopan

y exclusión, los cuales están asociados con la detonación de conflictos violentos. Finalmente, se tomó en consideración la ausencia de espacios recreativos y culturales dirigidos a la población joven, así como aspectos tales como la violencia en distintos espacios de socialización, la estigmatización a la que hacen frente los jóvenes pandilleros, y las experiencias de resiliencia experimentadas por actores socialmente reconocidos en las zonas bajo estudio, entre otros factores.

Finalmente, el primer capítulo expone algunos datos que sirvieron de base para la elaboración de las estrategias de intervención a implementar. Entre los más destacables se tiene que hay un total de 16522 hogares, cada uno de estos habitado, en promedio, por 4.4 personas. Es notable un alto porcentaje de población que ha migrado de otras entidades: dos de cada diez habitantes se encuentran en esta situación. Es destacable que en las cuatro colonias investigadas hay carencias importantes en materia de servicios públicos (es decir, pavimentación, electricidad, drenaje, etc.). Con respecto a la población ocupada puede decirse que en el 2010 , laboraba sólo el $34.3 \%$ de los habitantes de las colonias estudiadas ( $45 \%$ de estos en el sector secundario y $40 \%$ en el sector servicios; el resto se dedicaba a actividades vinculadas con el sector primario). A manera de contraste se tiene que cerca del $40 \%$ de los habitantes no cuenta con acceso a servicios de salud. Marcial y Vizcarra ponen de relieve que uno de los aspectos compartidos por las cuatro colonias tiene que ver con el marcado incremento de la deserción escolar, sobre todo en los niveles educativos posteriores a la secundaria. Como se observa, las zonas bajo estudio comparten situaciones de alta marginación, vulnerabilidad y conflictividad. Es por ello que en un contexto como el descrito resulta más que pertinente el esfuerzo realizado por los investigadores mencionados.

En el segundo capítulo, titulado "Discursos sobre el "pandillerismo': la mirada social sobre el fenómeno juvenil”, se 
efectúa una revisión crítica de los modos en que la sociedad percibe el fenómeno analizado. De manera particular, en este capítulo se abordan las perspectivas tanto de los medios de comunicación como de los vecinos de las colonias bajo análisis. Así, con respecto al primero de tales actores, es decir, los medios de comunicación, se tiene que estos consideran como causas del pandillerismo a factores tales como la ausencia de políticas públicas, la debilidad institucional para el combate a la violencia, la falta de espacios recreativos, el crecimiento urbano mal planeado, la desintegración familiar, la pobreza y la marginalidad. En este sentido, se observa que en los medios circula un discurso que postula a lo endeble de la arquitectura institucional como el núcleo alrededor del que surgen agrupaciones como las pandillas. De igual forma, de acuerdo con Marcial y Vizcarra, puede decirse que de entre las prácticas que llevan a cabo estas grupalidades, los medios de comunicación tienden a destacar dos: las de naturaleza delictiva (sobre todo homicidios, robos, riñas y consumo y posesión de sustancias ilegales), y aquellas de carácter identitario.

Ahora bien, ¿cuáles son algunos de los discursos articulados por los vecinos de las colonias analizadas? En principio, Marcial y Vizcarra señalan, en el segundo capítulo de su libro, que los habitantes de las zonas bajo estudio atribuyen la presencia de estos grupos a las condiciones de violencia y marginalidad que prevalecen en sus espacios cotidianos. Asimismo, el discurso de los habitantes de las zonas analizadas postula que los integrantes de las pandillas son cada vez más jóvenes. Se percibe que aun cuando algunos de los jóvenes integrantes de pandillas trabajan, muchos se dedican a delinquir (es decir, a robar). Lo anterior es atribuido a las condiciones precarias en las que se vive. En este sentido, del análisis realizado por Marcial y Vizcarra se desprende que por lo menos entre los vecinos se percibe la existencia de una especie de proceso de aprendizaje negativo, en el que el 
Porque así soy yo. Identidad, violencias y alternativas sociales entre jóvenes pertenecientes a "barrios" o "pandillas" en colonias conflictivas de Zapopan

contexto configura a los sujetos "pandilleros". En este punto vale la pena destacar, junto con los mencionados autores, que hay un incremento de la participación de las mujeres en tanto integrantes de las pandillas; es decir, estas emulan cada vez más a sus pares masculinos. Ello en lo que refiere tanto a las actividades "callejeras" como a la construcción de identidades vinculadas con los "grupos de esquina".

Aunado a lo anterior, Marcial y Vizcarra también descubrieron que los vecinos documentan la existencia del denominado pago de "derecho de piso". Esto es, una cuota mediante la que los pandilleros les permiten a los vecinos (y a figuras de autoridad, tales como los profesores de las escuelas de la zona) transitar y trabajar ahí sin ser molestados. En fin, el capítulo segundo ofrece tanto una mirada panorámica, de conjunto, con respecto a algunos factores compartidos por las cuatro colonias bajo estudio, como un abordaje acerca de las especificidades presentes en cada una de estas áreas. Con ello se pone de relieve que el escenario sobre el que Marcial y Vizcarra buscaron intervenir a partir de sus hallazgos se torna altamente complejo.

El tercer capítulo, titulado "El trabajo con los 'barrios' zapopanos", muestra algunos de los mecanismos implementados para la detección tanto de informantes clave que enriquecieran el estudio, como de jóvenes pertenecientes a pandillas capaces de convertirse en "actores de paz". De manera específica, una de las estrategias puestas en marcha por Marcial y Vizcarra consistió en la realización de eventos de esparcimiento, particularmente con actividades vinculadas con la música rap (concurso) y la exposición de perros de la raza conocida como pitbull. Lo que se buscaba en última instancia con esta estrategia era demostrar la posibilidad de asistir a una experiencia libre de violencia, en donde estuvieran pandillas de varios sectores de las colonias analizadas, algunas de ellas con conflictos fuertes entre sí. El objetivo central de estas "puestas en escena" 
consistía en localizar a jóvenes talentosos que pudieran, eventualmente, contribuir a la generación de productos culturales que tendieran a disminuir las prácticas de riesgo y que postularan alternativas para disminuir la violencia entre grupos rivales.

Marcial y Vizcarra aclaran que todo este proceso, desde la convocatoria a los eventos de esparcimiento hasta la participación de los integrantes de pandillas en las diversas actividades propuestas en la estrategia de intervención, fue registrado tanto en fotografía como en video (véase el DVD que acompaña al libro). De hecho, el material fue suficiente para producir dos documentales (largometrajes). El primero de estos muestra las actividades realizadas por el equipo de investigación conformado por los citados autores. Vale la pena mencionar que este es utilizado como parte del material que sirve de capacitación para el personal del área de Prevención del Delito, en Zapopan. El segundo de los largometrajes, titulado Donde moran los sueños, presenta los distintos puntos de vista con respecto al fenómeno investigado. De manera específica, en este se presentan las miradas de los jóvenes, de los vecinos, y también de las autoridades zapopanas. También, en este segundo producto audiovisual, se registran algunos aspectos cruciales de la vida cotidiana de quienes resultaron ganadores de los concursos de rap. Sobra decir que este material ha sido exhibido con éxito en diversos foros.

Todo lo anterior sirvió, además, de insumo para la estructuración de una campaña encaminada a la disminución de la violencia en el municipio. Esto adquiere una importancia vital puesto que está articulado alrededor de -tal como lo plantean Marcial y Vizcarra (2014) - la propia cosmovisión de los jóvenes, quienes "lanzan ese mensaje en contra de la violencia” (p. 96). En fin, no está de más señalar que este capítulo ofrece una sólida guía para el acercamiento a este tipo de grupalidades. Ello no solo en términos de metodologías y de diagnósticos, sino de procesos de intervención 
social replicables en realidades con características similares. Lo anterior con base en el abordaje de las miradas, la vida y las vicisitudes a las que se enfrenta este sector poblacional constituido en su mayoría por jóvenes. Se detalla, de manera específica, el conjunto de testimonios recabados; se hace una descripción del perfil sociodemográfico de la población bajo estudio, de las experiencias y las relaciones que sostienen los integrantes de estos grupos con la esfera institucional, del modo en que distribuyen su tiempo; las actividades que realizan cuando están junto con la pandilla; las sustancias ilícitas que consumen; los referentes identitarios que suscriben (es decir, la música rap); sus experiencias en un entorno marcado por la violencia. En fin, el trabajo realizado en este capítulo por Marcial y Vizcarra constituye, sin duda, uno de los grandes aportes de esta obra.

Por último, el cuarto capítulo se titula "Identidad y violencias sociales". En este, Marcial y Vizcarra postulan que para entender el contexto de algunas prácticas sancionadas socialmente como violentas se precisa comprender el incremento de la violencia que atraviesa al país en general. Los autores mencionan como un factor significativo de lo anterior la denominada "guerra contra el crimen organizado", iniciada durante el mandato del entonces presidente Felipe Calderón. Para documentar este tema, enumeran algunos rasgos que demuestran que la violencia en el país tiene en los jóvenes tanto a las víctimas como a los victimarios: la tasa de homicidios se ha incrementado más del triple. Aún cuando este flagelo afecta más a los hombres, Marcial y Vizcarra encuentran que el número de víctimas femeninas va en aumento. Como quiera que sea, los mencionados autores plantean que ello se debe, en buena medida, al modelo económico prevaleciente en el país, el cual ha tenido como consecuencia la ruptura del tejido social. Lo anterior genera escenarios marcados por la incertidumbre, en los que la solidaridad se erosiona, y los esquemas de inserción social tiende a interrumpirse: 
el tránsito por la institución escolar ya no le garantiza a la juventud la consecución de un salario digno que permita emanciparse y formar un nuevo núcleo familiar.

En este contexto, Marcial y Vizcarra plantean que con respecto a las pandillas que habitan el municipio de Zapopan es posible observar algunas tendencias que prevalecen hasta nuestros días. Así, en principio, los citados autores argumentan que la principal causa para incorporarse a estas agrupaciones tiene que ver con que estar fuera de estas constituye un riesgo para la integridad física. En este mismo sentido, se observa que las formas violentas que adquieren los modos de expresión de estos grupos están asociadas con la disputa por el territorio. Es precisamente este uno de los aspectos que permanece: la construcción de una violencia simbólica, nucleada alrededor de la competencia por ver quién es el mejor, el más duro, el más "machín”. No obstante, de manera reciente, se ha observado un desplazamiento de la violencia simbólica hacia planos más reales, en donde la fuerza no sólo se representa, sino que se demuestra en enfrentamientos físicos que involucran desde los puños hasta armas blancas y armas de fuego. En este contexto, Marcial y Vizcarra encuentran que hay un proceso de erosionamiento institucional. Así, instancias como la escuela han perdido la importancia que solían tener en tanto espacios cruciales para la socialización. En este mismo sentido, la ciudad tampoco constituye un referente para este proceso. Incluso, entre los jóvenes adscritos a pandillas, hay ocasiones en que el propio barrio es percibido como inseguro. Hay en todo lo anterior una evidente falta de sentido percibida por este sector poblacional. Tal como lo señalan los mencionados autores, existe la sensación de que no hay un proyecto a futuro; ni una realidad positiva en el presente. Frente a ello, el posicionamiento por el que opta buena parte de los jóvenes pandilleros se sitúa en la necesidad de vivir con alta intensidad su juventud. Ello, desde luego, está aso- 
Porque así soy yo. Identidad, violencias y alternativas sociales entre jóvenes pertenecientes a "barrios" o "pandillas" en colonias conflictivas de Zapopan

ciado con la puesta en marcha de prácticas de riesgo y con los incrementos de la violencia. Se genera así una especie de espiral descendente, de desencantos, cuyo desmontaje requiere de la implementación de estrategias innovadoras que, por un lado, produzcan información pertinente y, por otro, la utilicen para transformar positivamente la realidad. A lo largo del texto escrito por Marcial y Vizcarra queda claro que esa es la apuesta que se hace.

En fin, hasta aquí se describió de manera breve el contenido de los cuatro capítulos que le dan cuerpo al documento producido por Marcial y Vizcarra. En la parte última del texto, la dedicada a las conclusiones, los mencionados autores plantean la necesidad de tener una comprensión más profunda tanto de la violencia en general, como de las expresiones particulares que esta adopta cuando se aborda desde las miradas de las juventudes involucradas en pandillas. Lo anterior tiene como objetivo la postulación de una apuesta que se despliega tanto en el plano ético como el estético. Se plantea, específicamente, a la cultura como una posible vía para la reconstrucción del tejido social. Esto es así porque, tal como lo argumentan los mencionados autores, buena parte de lo que ocurre con las juventudes contemporáneas tiene que ver con la producción, distribución y naturalización de construcciones discursivas que estigmatizan a ciertos sectores de la juventud (las pandillas, por ejemplo). En este sentido, la estrategia central sobre la que Marcial y Vizcarra desarrollan sus argumentos, plantea que es preciso posicionar otro discurso. Este, proveniente del ámbito académico, debería anteponerse a discursos falaces, tendenciosos. No cabe duda que, quizá como un resultado inesperado, la obra producida por Marcial y Vizcarra constituirá, sin duda, una especie de prueba de ácido que permitirá tomar el pulso al gobierno municipal zapopano, el cual, dicho sea de paso, recientemente ha tenido diversos dislates en lo que refiere a la supuesta atención que le brinda a sus juventudes. 